\title{
A.M. Ripellino, Lo splendido violino verde, ed. introdotta e commentata da U. Bru- netti, con due scritti di C. Bologna e A. Fo, Artemide, Roma 2021, pp. 30 I.
}

Dal Barocco a Gozzano, dai crepuscolari all'espressionismo e al futurismo, arrivando fino a Montale l'incedere poetico di Angelo Maria Ripellino sembra muoversi in un territorio composito e pieno di insidie per chi ne voglia affrontare una lettura critica. Si tratta di versi caratterizzati da una amalgama lirica capace di assorbire ogni tipo di suggestione artistica, ma che nasconde sempre, quale doppiofondo, il sé riflesso del poeta. Perché i versi de Lo splendido violino verde, forse l'apice della produzione di Ripellino, sono un colloquio continuo con la propria anima, con la sofferenza dell'essere, con la malattia che si tramuta quotidianamente in male di vivere, ma che è anche lo specchio attraverso il quale filtrare la propria visione dell'esistenza.

Quasi penalizzato dalla sua attività di critico e cultore delle letterature slave, come lui stesso lamentava ('Slavista! Mi gridano donne con frappe sul capo'), il poeta Ripellino è stato visto e viene forse ancora percepito come un'appendice, magari a volte anche un po' troppo enfatica, del traduttore innamorato e divertito, filologo ineccepibile, del saggista dalla prosa creativa, capace di fantastiche esibizioni da circo, quasi fosse una sorta di trapezista della scrittura, un equilibrista sempre in bilico tra mondi artistici diversi.

Grazie a questa edizione critica curata da Umberto Brunetti possiamo invece renderci conto di come sia vero il contrario e Brunetti stesso lo afferma con decisione: prima di tutto Ripellino è un poeta ed è da questa vocazione, verrebbe da dire senza appello, che scaturisce lo studioso, l'appassionato cultore di tutte le letterature, il fascinoso affabulatore, l'autore di un' incredibile messe di critiche letterarie e teatrali. Anche per questo Ripellino occupa un posto centrale nella vita letteraria e nella poesia italiana del secondo Novecento. Con un portato di novità lessicali e ritmiche che ha pochi contendenti.

Corrado Bologna nella sua bella prefazione al volume ci fornisce chiavi di lettura molto acute e interessanti per avvicinarci alla comprensione di questi versi, così ricchi di sottotesti, di richiami letterari, alimentati dalla cultura ripelliana, che per molti di noi si identifica tout court con la cultura. Perché chi è stato suo allievo, magari anche per il pochissimo tempo che, ad esempio, fu a me concesso dall'orribile chiasso della storia alla fine degli anni Settanta del secolo scorso e dalla malattia che lo stroncò, nutriva non il sospetto, ma la certezza che Ripellino sapesse tutto di musica, di pittura, di teatro e di letteratura. Tutto, ma proprio tutto.

I versi di Ripellino sono fortemente incastonati in un determinato periodo storico, caratterizzato dal boom economico e dalla guerra fredda, dalla contestazione giovanile, in Italia anche dalla 
strategia della tensione e dal terrorismo e al tempo stesso si muovono in un mondo reinventato, popolato di personaggi letterari e teatrali, nella vita parallela che ci regalano la letteratura e l'arte per chi voglia avventurarsi in quell'itinerario nel meraviglioso, titolo ma anche contenuto di una sua famosa raccolta di saggi. Lungi dall'essere un poeta laureato Ripellino assimila, metabolizza il presente storico e lo mescola con la sua vicenda personale e con il materiale letterario, creando una scrittura originale, ricca di vocaboli desueti, di parole straniere, ma anche non scevra dall'uso del linguaggio comune. La sua poesia percepisce il crepuscolo, si balocca con il barocco, non disdegna i giochi futuristici, ma il fasto è solo apparente. $\grave{E}$ una poesia colta, certo, ma non aristocratica. Di sicuro la lingua, lo stile sono anche debitori ai tanti poeti russi e cechi che Ripellino ha tradotto e reinterpretato. Ma la sua straordinaria originalità è nell'utilizzare queste reminiscenze creando un pastiche linguistico/musicale senza eguali. A me pare, inoltre, che, nella volontà totalizzante di abbracciare tutto l'universo percepibile, la lirica di Ripellino sia anche definibile come poesia civile: il suo è uno sguardo costante sul mondo, di denuncia, di rimpianto non per un passato felice quanto per un impossibile futuro. In questo senso la sua lirica, il suo splendido violino, suona come un grido di allarme, come il tentativo di chiamare a raccolta quanti, pochi, troppo pochi, sentono come lui l'urgenza, la necessità di stabilire un'equivalenza tra vita e letteratura, l'una nell'altra confuse.

Così come Pasternak riteneva il Dottor Živago la sua unica opera veramente compiuta, Ripellino non fa mistero di sentirsi poeta e di volere essere riconosciuto come tale, non di certo per ostentare la fama di un premio letterario, quanto per il desiderio di venire apprezzato come uomo.

Scrive Bologna che: "Con questa edizione, la prima commentata di una sua raccolta di versi, quarant'anni dopo la scomparsa che tutti portiamo tagliente nel cuore, memoria d'una ferita terribile (1978), Ripellino entra finalmente nel mondo dei Classici, come ha sempre meritato e come pochi poeti contemporanei hanno ottenuto" (p. ir) e sottolinea come: "Il lettore troverà nelle pagine che seguono un fenomenale apparato ermeneutico, rigoroso, sensibilissimo, che si fonda su uno studio insieme accanito e innamorato" (p. 22). Alla prefazione di Bologna segue l'Introduzione di Brunetti che fa il punto sullo stato degli studi dedicati alla poesia di Ripellino, ma ci offre anche delle riflessioni che sommate a quelle di Bologna, danno un quadro molto ampio delle possibili chiavi interpretative de Lo splendido violino verde, tanto da poterci portare a sostenere che si tratta di un'analisi davvero esaustiva per la profondità delle considerazioni e degli spunti critici offerti. Segue la pubblicazione dell' intera raccolta che consta di 86 poesie composte tra il 1972 e il 1975 . Ciascuna lirica è preceduta da un breve scritto introduttivo che ne spiega il carattere e il contenuto. Corredano ogni testo delle accuratissime note che ci consentono di penetrare appieno il significato e i possibili rimandi di ogni verso, un "fenomenale apparato" che: "mira a districare la vasta eterogeneità di influssi culturali presenti nelle liriche di Ripellino e approdare a una visione più definita di questa voce poetica non ordinaria, che meriterebbe di trovare posto nel canone del nostro secondo Novecento” (p. 39).

Il volume è chiuso da uno scritto di Alessandro Fo che rievoca la sua casuale scoperta de Lo splendido volume verde: un ricordo personale che, inevitabilmente, così come pure accade nella prefazione di Corrado Bologna, si colora di accenti commossi. Non so se Ripellino si possa definire come "poeta del rimpianto", ma certamente è questo il sentimento che attraversa quanti, anche grazie a questo volume curato da Umberto Brunetti, sono sicuri di poterlo celebrare come una delle voci liriche più alte del Novecento. 